\title{
Conflict between the identification of cosmic neutrino source and the sensitivity to mixing angles in neutrino telescope
}

\author{
Ggyoung-Riun Hwang* and Kim Siyeont \\ Department of Physics, Chung-Ang University, Seoul 156-756, Korea
}

\begin{abstract}
Neutrino fluxes at telescopes depend on both initial fluxes out of astronomical bursts and flavor mixing during their travel to the earth. However, since the information on the initial composition requires better precision in mixing angles and vice versa, the neutrino detection at telescopes for itself cannot provide solutions to the both problems. Thus, a probability to be measured at long baseline oscillation is considered as a complement to the telescope, and problems like source identification and parameter degeneracy are examined under a few assumptions.
\end{abstract}

PACS numbers: 14.60.Pq, 95.55.Vj, 98.70.Sa

*Electronic address: galaraja@phys.cau.ac.kr

$\dagger$ Electronic address: siyeon@cau.ac.kr 


\section{INTRODUCTION}

Cosmic neutrinos can be classified into stellar, galactic, and extra-galactic neutrinos according to astronomical source. Stellar neutrinos include solar and supernova neutrinos, of which energy scale is order of (1-10) MeV since they are mainly produced by nuclear interaction. There are fair records in which they were identified as from the sun and SN1987A and examined for oscillation and matter effect [1] [2]. Ultrahigh energy (UHE; $\gtrsim 10^{18} \mathrm{eV}$ ) cosmic particles are regarded to have their origin in extra-galactic source like active galactic nuclei(AGN) and some of gamma ray bursts(GRB). Mechanisms to accelerate protons to high energy have been searched, from $\mathcal{O}(\mathrm{PeV})$ to GZK limit, in GRB [3] [4]. In astronomy, GRB's are detected once a day on average. If an accelerated proton produces a pion, about $20 \%$ of its energy is transferred to neutrinos, although more than one pion can be produced from a proton. Thus there are abundant source of neutrinos whose energy is higher than $100 \mathrm{TeV}[5]$.

Decays of pions, such as $\pi^{+} \rightarrow \mu^{+}+\nu_{\mu} \rightarrow e^{+}+\nu_{\mu}+\nu_{e}+\bar{\nu}_{\mu}$ or $\pi^{-} \rightarrow \mu^{-}+\bar{\nu}_{\mu} \rightarrow$ $e^{-}+\nu_{\mu}+\bar{\nu}_{e}+\bar{\nu}_{\mu}$, are the main process of neutrino production. The initial neutrino flavor ratio can be determined, depending on the charges of pions and the energy loss rates of muons in processes of pion decays. Whether pions are produced by $p p$ or by $p \gamma$ collision, the charges of pions and the ratio of $\nu_{e} / \bar{\nu}_{e}$ are different. Although both pions and muons lose energy in environment that pions were borne, long-lived muons are more probable to interact with environment before decaying. The pions that decay into neutrinos without muon decays are called "muon-damped source". Such electromagnetic energy loss of muons for GRB becomes significant when the energy of $\gamma$-ray $E_{\gamma}$ is $\gtrsim 100 \mathrm{TeV}$ and, thus, the

energy of neutrino $E_{\nu}$ produced from muon-damped source is $\gtrsim 1 \mathrm{TeV}[6]$. The intensities of neutrinos in $10^{14} \mathrm{eV}<\mathrm{E}<10^{16} \mathrm{eV}$ and higher energy were studied. Its model-independent upper bound was predicted [4] [7]. If the decay mode of a pion includes muon decay, that will be called simply "pion source" in comparison with muon-damped source. The initial neutrino composition $\Phi^{0}\left(\nu_{e}\right): \Phi^{0}\left(\nu_{\mu}\right): \Phi^{0}\left(\nu_{\tau}\right)=1: 2: 0$ from the pion source is gradually replaced by the composition $\Phi^{0}\left(\nu_{e}\right): \Phi^{0}\left(\nu_{\mu}\right): \Phi^{0}\left(\nu_{\tau}\right)=0: 1: 0$ from the muon-damped source when the energy increases passing about $1 \mathrm{TeV}$ [6] [8]. When the main source of neutrino production in atmosphere is pion decay, the shift of the ratio $\Phi^{0}\left(\nu_{\mu}\right) / \Phi^{0}\left(\nu_{e}\right)$ from 2 to infinity according to increasing energy [9] [10] was tested in SK [11][12]. The transition 
in the flavor ratio can be naturally assumed to occur at the region of GRB's, and the corresponding aspect was discussed in Ref. [6]. The relative flavor ratios of detected fluxes vs. energy exhibit saturation and transition. The saturation of $\Phi^{t}\left(\nu_{\mu}\right) / \Phi^{t}\left(\nu_{e}\right)$ close to one implies that the beam originated from pion source for lower energy, while the transition of $\Phi^{t}\left(\nu_{\mu}\right) / \Phi^{t}\left(\nu_{e}\right)$ to a larger one implies that the beam includes portion of muon-damped source for higher energy.

There are a few neutrino telescopes under construction, which can detect high energy neutrinos of $E_{\nu}>0.1 \mathrm{TeV}$, e.g., IceCube [13], Antares [14], etc. They are designed to detect VHE and UHE neutrinos and to distinguish a flavor from others. With a future Cerenkov detector, $\nu_{\mu}$ is easy to be detected due to the long tracks of muons, while $\nu_{\tau}$ is distinguishable from $\nu_{e}$ by double-bang event only near PeV range. At $\mathcal{O}(\mathrm{PeV})$, the neutrino telescopes will be able to observe both double showers of $\nu_{\tau}$, one due to $\tau$ production and the other due to $\tau$ decay, and $W^{-}$resonant event at $6.3 \mathrm{PeV}$ that identifies $\bar{\nu}_{e}[15]$.

For cosmic neutrinos, the oscillation factor in a transition probability is averaged out due to long distance of travel and high frequency. Thus, the telescope experiment can be effective measurements of mixing parameters, provided that the neutrino flavor ratio at the source is known [16] The current data of mixing parameters are phrased by the ranges in the magnitude of PMNS elements;

$$
\left|U_{P M N S}\right|=\left(\begin{array}{ccc}
0.79-0.86 & 0.50-0.61 & 0-0.20 \\
0.25-0.53 & 0.47-0.73 & 0.56-0.79 \\
0.21-0.51 & 0.42-0.69 & 0.61-0.83
\end{array}\right)
$$

at $3 \sigma$ level [17]. The matrix implies the following values of individual parameters; $\Delta m_{21}^{2}=$ $(7.1-8.9) \times 10^{-5} \mathrm{eV}^{2},\left|\Delta m_{31}^{2}\right|=(2.2-3.0) \times 10^{-3} \mathrm{eV}^{2}, \quad \sin ^{2} \theta_{12}=0.24-0.40, \quad \sin ^{2} \theta_{23}=$ $0.34-0.68$, and $\sin ^{2} \theta_{13} \leq 0.040$, all at $3 \sigma$ level [18]. The type of the yet-undetermined among neutrino parameters can be organized case by case: First, normal hierarchy (NH) or inverse hierarchy (IH): the sign of $\Delta m_{31}^{2} \equiv m_{3}^{2}-m_{1}^{2}$ is unknown while the global best-fit is obtained as $\left|\Delta m_{31}^{2}\right|=2.6 \times 10^{-3} \mathrm{eV}^{2}$. Second, the sign of $\theta_{23}-\pi / 4$, i.e., whether the majority of $\nu_{\tau}$ is the heavier $\left(\nu_{3}\right.$ for $\left.\mathrm{NH}\right)$ or the $\operatorname{lighter}\left(\nu_{2}\right.$ for $\left.\mathrm{NH}\right)$ is unknown. Third, a certain value of oscillation probability has infinite number of candidate combination of $\theta_{13}$ and $\delta_{C P}$ [19] [20]. The ambiguity due to the above degeneracies needs to be clearly distinguished from that due to uncertainties. 
In Sec. II, the probability of a neutrino oscillation is reviewed, focused on how the degeneracies are generated and which oscillation experiment each mixing angle is most sensitive to. In Sec. III, examined are the sensitivities of neutrino fluxes to neutrino mixing angles. In Sec. IV, we discussed a few points including parameter degeneracies and source identification, where the data of neutrino telescopes are considered in company with the data of a long baseline (LBL) oscillation under a few assumptions. Concluding remarks follow in Sec. V

\section{REVIEW ON DEGENERATE PROBABILITY OF OSCILLATION}

The probability of neutrino oscillation may be said to be degenerate because different sets of parameters result in the same value. For example, the probability of transition from $\nu_{\alpha}$ to $\nu_{\beta}$ in two-neutrino oscillation with a single mixing angle and a single mass-squared difference $\Delta m^{2}$

$$
P_{\alpha \beta}=\delta_{\alpha \beta}-\left(2 \delta_{\alpha \beta}-1\right) \sin ^{2} 2 \theta \sin ^{2}\left(\frac{\Delta m^{2} L}{4 E}\right)
$$

is invariant under switching the sign of $\Delta m^{2}$ or changing the angle $\theta$ with its complementarity angle $\pi / 2-\theta$. Since the two sets, $\left(\theta, \Delta m^{2}\right)$ and $\left(\pi / 2-\theta,-\Delta m^{2}\right)$, are not physically different, the above probability is twofold degenerate simply due to $\left(\theta, \Delta m^{2}\right)$ and $\left(\pi / 2-\theta, \Delta m^{2}\right)$ or due to $\left(\theta, \Delta m^{2}\right)$ and $\left(\theta,-\Delta m^{2}\right)$.

The oscillation probability extended to three neutrinos in vacuum

$$
\begin{aligned}
P_{\alpha \beta}=\delta_{\alpha \beta} & -4 \sum_{i=1}^{2} \sum_{j=i+1}^{3} \operatorname{Re}\left[U_{\alpha i} U_{\beta i}^{*} U_{\alpha j}^{*} U_{\beta j}\right] \sin ^{2}\left(\frac{\Delta m_{j i}^{2} L}{4 E}\right) \\
& \pm 2 \sum_{i=1}^{2} \sum_{j=i+1}^{3} \operatorname{Im}\left[U_{\alpha i} U_{\beta i}^{*} U_{\alpha j}^{*} U_{\beta j}\right] \sin \left(\frac{\Delta m_{j i}^{2} L}{2 E}\right)
\end{aligned}
$$

is given in terms of $3 \times 3$ unitary transformation matrix $U$ and three mass-squared differences $\Delta m_{j i}^{2} \equiv m_{j}^{2}-m_{i}^{2}$, where each of $\nu_{\alpha}$ and $\nu_{\beta}$ may be one of $\nu_{e}, \nu_{\mu}$ or $\nu_{\tau}$. In effect $\Delta m_{31}^{2} \simeq$ $\Delta m_{32}^{2}$ so that they will not be distinguished hereafter. The PMNS matrix in standard parametrization is given by

$$
U_{\alpha i} \equiv\left(\begin{array}{ccc}
c_{12} c_{13} & s_{12} c_{13} & s_{13} e^{-i \delta} \\
-s_{12} c_{23}-c_{12} s_{23} s_{13} e^{i \delta} & c_{12} c_{23}-s_{12} s_{23} s_{13} e^{i \delta} & s_{23} c_{13} \\
s_{12} s_{23}-c_{12} c_{23} s_{13} e^{i \delta} & -c_{12} s_{23}-s_{12} c_{23} s_{13} e^{i \delta} & c_{23} c_{13}
\end{array}\right)
$$


where $s_{i j}$ and $c_{i j}$ denote $\sin \theta_{i j}$ and $\cos \theta_{i j}$ with the mixing angle $\theta_{i j}$ between $i$-th and $j$-th generations, respectively, and $\delta$ denotes a Dirac phase.

The degeneracy of probability could be stemmed from three mixing angles and three mass-squared differences. However, since a number of successful experiments found the values of some physical parameters, the possible multiplicity of degeneracy at present is at most eight. A twofold degeneracy in the eightfold degeneracy has its origin in the ambiguity of a value of $\theta_{23}$ from its complementary angle, $\pi / 2-\theta_{23}$. The $\nu_{\mu}$-disappearance probability which is most sensitive to determine the value of $\theta_{23}$ is expressed by [20]

$$
\begin{aligned}
& 1-P\left(\nu_{\mu} \rightarrow \nu_{\mu}\right)=\sin ^{2} 2 \theta_{23} \cos ^{2} \theta_{13} \sin ^{2}\left(\frac{\Delta m_{31}^{2} L}{4 E_{\nu}}\right) \\
& -\sin \left(\frac{\Delta m_{21}^{2} L}{4 E_{\nu}}\right) \sin \left(\frac{\Delta m_{31}^{2} L}{4 E_{\nu}}\right) \sin ^{2} 2 \theta_{23} . \\
& \cdot\left(\cos ^{2} \theta_{13} \cos ^{2} \theta_{12}-\sin \theta_{13} \sin ^{2} \theta_{23} \sin 2 \theta_{12} \cos \delta\right),
\end{aligned}
$$

where the leading two terms are invariant under the exchange of $\theta_{23}$ with its complementary angle. On the other hand, the $\nu_{e}$-appearance probability with matter effect $A$,

$$
\begin{aligned}
& P\left(\nu_{\mu}\left(\bar{\nu}_{\mu}\right) \rightarrow \nu_{e}\left(\bar{\nu}_{e}\right)\right)=\sin ^{2} \theta_{23} \sin ^{2} 2 \theta_{13} \frac{\sin ^{2}\left(\left(\Delta m_{31}^{2} \mp A\right) L / 4 E_{\nu}\right)}{\left(1 \mp A / \Delta m_{31}^{2}\right)^{2}} \\
& +\frac{\Delta m_{21}^{2}}{\Delta m_{31}^{2}} \sin 2 \theta_{23} \sin 2 \theta_{13} \sin 2 \theta_{12} \cos \left(\delta \pm \Delta m_{31}^{2} L / 4 E_{\nu}\right) \cdot \\
& \quad \frac{\sin \left(\left(\Delta m_{31}^{2} \mp A\right) L / 4 E\right) \sin \left(A L / 4 E_{\nu}\right)}{\left(1 \mp A / \Delta m_{31}^{2}\right)\left(A / \Delta m_{31}^{2}\right)} \\
& +\left(\frac{\Delta m_{21}^{2}}{\Delta m_{31}^{2}}\right)^{2} \cos ^{2} \theta_{23} \sin ^{2} 2 \theta_{12} \frac{\sin ^{2}\left(A / 4 E_{\nu}\right)}{\left(A / \Delta m_{31}^{2}\right)^{2}}
\end{aligned}
$$

provides multi solutions to $\left(\theta_{13}, \delta\right)$ pair and $\left(\Delta m_{31}^{2}, \delta\right)$ pair, where $A \equiv 2 \sqrt{2} \mathrm{G}_{\mathrm{F}} \mathrm{Y}_{\mathrm{e}} \rho E_{\nu}$ is given with a density $\rho$ and an electron fraction $Y_{e}[19][20]$. The leading term in Eq. (5) is not sensitive to the sign of $\Delta m_{31}^{2}$, if the matter effect $\mathrm{A}$ is not significant. The second term is also not indicative of the sign due to $\cos \delta$ and a suppressing factor $\Delta m_{21}^{2} / \Delta m_{31}^{2}$. So the $\left(\Delta m_{31}^{2}, \delta\right)$ pair causes another double degeneracy to the probability. It is well known as a matter of normal hierarchy or inverse hierarchy. The multiple possibilities in the pair of $\left(\theta_{13}, \delta\right)$ for a value of $\sin 2 \theta_{13} \cos \delta$ make up the eight-fold degeneracy with other two double degeneracies.

If astronomical neutrinos from origins like grb's or other types of extragalactic bursts are considered for the flavor transition, in the limit $L \rightarrow \infty$, the probability in Eq. (3) reduces 
to

$$
\begin{aligned}
P_{\alpha \beta}^{t} & \rightarrow \delta_{\alpha \beta}-2 \sum_{i=1}^{2} \sum_{j=i+1}^{3} \operatorname{Re}\left[U_{\alpha i} U_{\beta i}^{*} U_{\alpha j}^{*} U_{\beta j}\right] \\
& =\sum_{i=1}^{3}\left|U_{\alpha i}\right|^{2}\left|U_{\beta i}\right|^{2} .
\end{aligned}
$$

Since the probability of relatively long-distance oscillation compared to wavelength is averaged out, the dependency in the sign of $\Delta m_{j i}^{2}$ is hidden, so that the probability is blind to $\Delta m_{j i}^{2}$. Since it depends on only the absolute values of the elements in PMNS as shown in Eq. (17), its dependency on the phase $\delta$ is simply $\cos \delta$. Sensitive dependencies between other mixing angles and $P_{\alpha \beta}^{t}$ will be discussed in detail in Section IV.

Degeneracy problems are caused since the number of effective measurements is not sufficient to specify all the physical parameters and the forms of probabilities are multi-variable sinusoidal functions. Here, the ambiguities from three types of degeneracies, the sign of

$\Delta m_{31}^{2}$ [21], the sign of $\pi / 4-\theta_{23}$ [22], and different pairs of $\left(\theta_{13}, \delta\right)$ 's [23] will be clearly distinguished from the ambiguities from uncertainties. The degeneracy is a problem causing ambiguity even when an average probability or an average flux without uncertainty is applied to determine parameters. For more review, see references [24].

\section{LIMIT OF NEUTRINO TELESCOPE}

The initial flux of astronomical high energy neutrinos is assumed to be attributed from the decay of pion produced in $p \gamma$ collision. The relative ratio of neutrino flavors from pion decay is $\Phi^{0}\left(\nu_{e}\right): \Phi^{0}\left(\nu_{\mu}\right): \Phi^{0}\left(\nu_{\tau}\right)=1: 2: 0$. If the daughter muon in pion decay does not decay due to the electromagnetic energy loss, the flavor ratio is changed into $\Phi^{0}\left(\nu_{e}\right): \Phi^{0}\left(\nu_{\mu}\right): \Phi^{0}\left(\nu_{\tau}\right)=0: 1: 0$. So, the composition ratio $\Phi^{0}\left(\nu_{\mu}\right) / \Phi^{0}\left(\nu_{e}\right)=2$ and its transition to infinity as the energy of neutrinos increases may occur in region of GRB's, as does in atmosphere. However, it is impossible to estimate the initial flux of neutrinos as being produced in GRB's. One can but conjecture it from detected fluxes at telescopes [25]. If the neutrino mixing is of tri-bi maximal type as the simplest example, the pion source is identified by the flux ratio at telescopes, $\Phi^{t}\left(\nu_{e}\right): \Phi^{t}\left(\nu_{\mu}\right): \Phi^{t}\left(\nu_{\tau}\right)=1: 1: 1$, while the muondamped source is identified by the flux ratio at telescopes, $1: 1.8: 1.8$. Thus, the transition in flavor ratios of detected fluxes at GRB's occurs with the relative ratio $\Phi^{t}\left(\nu_{\mu}\right) / \Phi^{t}\left(\nu_{e}\right)$ from 1 
to 1.8 as the energy of neutrinos increases. In reality, it is impossible to determine, simply by reading results at telescopes, whether initial beams are pion source or muon-damped source, without any assumption, for the following reasons: First, any information from astronomical neutrino bursts on the composition in a mixture of two types of sources cannot be obtained without using telescopes. Second, the mixing angles and masses are still bearing too broad uncertainties to analyze the fluxes, and moreover the fluxes to be detected at telescopes are significantly sensitive to mixing angles. Hereafter, we took a strong assumption which is that pion source and muon-damped source can be distinguished, in order to illustrate the point of the sensitivity to mixing angles.

Unless neutrinos decay, $\Phi^{0}\left(\nu_{e}\right)+\Phi^{0}\left(\nu_{\mu}\right)+\Phi^{0}\left(\nu_{\tau}\right)=\Phi^{t}\left(\nu_{e}\right)+\Phi^{t}\left(\nu_{\mu}\right)+\Phi^{t}\left(\nu_{\tau}\right)$ and $\sum_{\alpha} P_{\alpha \beta}^{t}=$ 1 , where $\Phi^{t}\left(\nu_{\beta}\right)=\sum_{\alpha} P_{\alpha \beta}^{t} \Phi^{0}\left(\nu_{\alpha}\right)$. Since relative fluxes at a telescope can be normalized such that $\sum_{\alpha} \Phi^{0}\left(\nu_{\alpha}\right)=\sum_{\alpha} \Phi^{t}\left(\nu_{\alpha}\right)=1$, the normalized flux $\Phi^{t}\left(\nu_{\alpha}\right)$ is a linear combination of $P_{\beta \alpha}^{t}$ 's, for example, $\Phi^{t}\left(\nu_{\alpha}\right)=P_{\mu \alpha}^{t}$ for muon-damped source and $\Phi^{t}\left(\nu_{\alpha}\right)=1 / 3\left(P_{e \alpha}^{t}+2 P_{\mu \alpha}^{t}\right)$ for pion source. The symmetric matrix $P_{\alpha \beta}^{t}$ in Eq. (7) has only three independent elements. Thus, when the initial condition of neutrino beam is assumed such that both pure pion source and pure muon-damped source are allowed, $\Phi^{t}\left(\nu_{\tau}\right)$ and one of the following four fluxes may be unnecessary: $\Phi^{t}\left(\nu_{e}\right)$ from pion source, $\Phi^{t}\left(\nu_{\mu}\right)$ from pion source, $\Phi^{t}\left(\nu_{e}\right)$ from muon-damped source, and $\Phi^{t}\left(\nu_{\mu}\right)$ from muon-damped source. In reality, either $\Phi^{t}\left(\nu_{e}\right)$ or $\Phi^{t}\left(\nu_{\mu}\right)$ is more likely to be a mixed flux from pion source and muon-damped source, rather than a flux from the pure pion source or the pure muon-damped source. In order to examine the sensitivities to mixing angles, however, we consider the pure pion or the pure muon-damped source. FIG. 1 and FIG. 2 illustrate the dependence of $\Phi^{t}\left(\nu_{e}\right)$ on $\theta_{23}$ and the dependence of $\Phi^{t}\left(\nu_{\mu}\right)$ on $\theta_{23}$, respectively. For a given $\theta_{13}$, the range of $\delta$ from 0 to $2 \pi$ makes the curves thick bands. The red (upper in Fig. 1 and lower in Fig.2)and blue (lower in Fig.2 and upper in Fig.1) bands indicate the fluxes from the pure pion source or from the pure muon-damped source, respectively.

The symmetric probability in Eq. (7) has ranges estimated from PMNS at $3 \sigma$ CL in Eq. (1);

$$
P_{\alpha \beta}=\left(\begin{array}{ccc}
0.48-0.64 & 0.12-0.34 & 0.11-0.35 \\
\sqrt{ } & 0.33-0.53 & 0.30-0.41 \\
\sqrt{ } & \sqrt{ } & 0.33-0.47
\end{array}\right) .
$$

Thus, the fluxes accompany nontrivial ranges. The plots in FIG. 11 and FIG. 2 illustrate the 

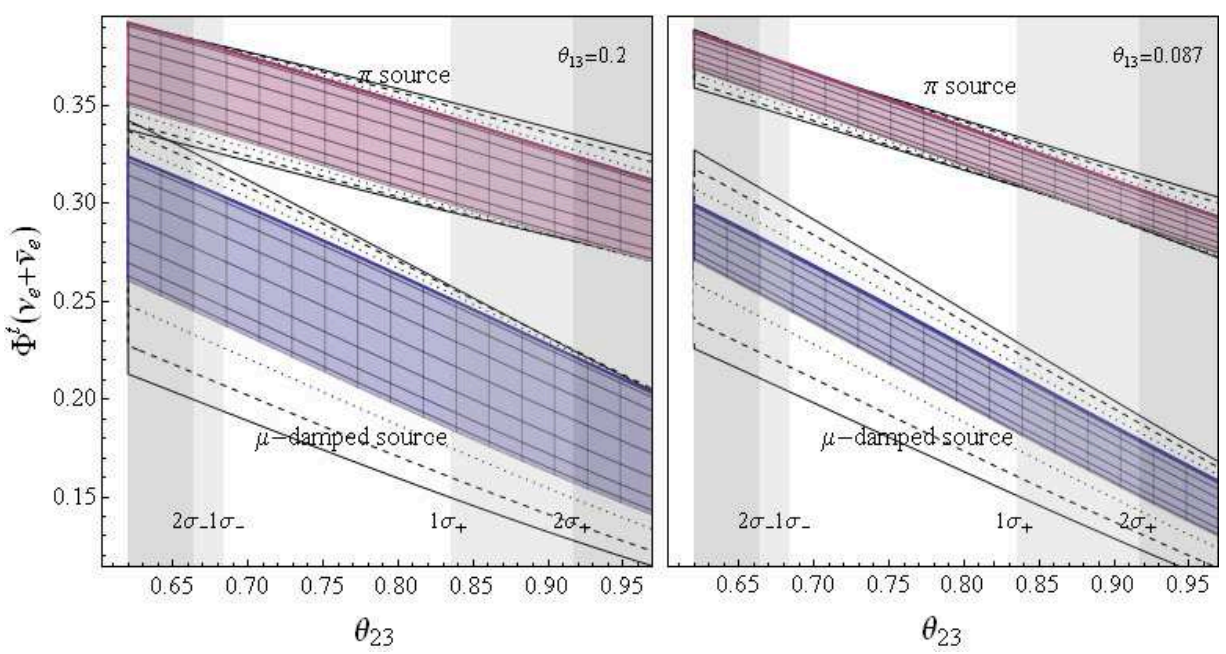

FIG. 1: $\Phi^{t}\left(\nu_{e}\right)$ vs. $\theta_{23}$ for fixed values of $\theta_{13}$.
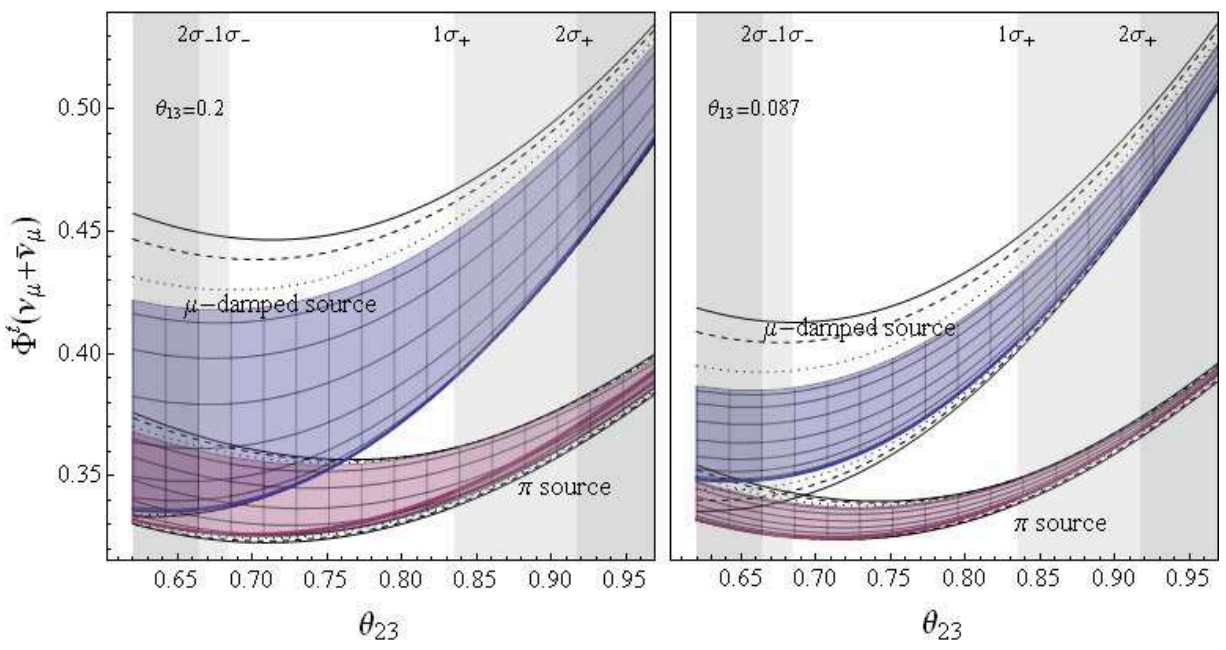

FIG. 2: $\Phi^{t}\left(\nu_{\mu}\right)$ vs. $\theta_{23}$ for fixed values of $\theta_{13}$.

sensitivity of fluxes to all the mixing angles including CP phase $\delta$. The significant sensitivity to $\theta_{23}$ is expressed by the broad coverage in $\Phi^{t}\left(\nu_{e}\right)$ and $\Phi^{t}\left(\nu_{\mu}\right)$ of each colored stripe for fixed $\theta_{12}$ and $\theta_{13}$. Each stripe is a bundle of curves for $\delta$ values from 0 to $\pi$. The top line of the stripe for $\Phi^{t}\left(\nu_{e}\right)$ indicates $\delta=0$, while the bottom line of the stripe for $\Phi^{t}\left(\nu_{\mu}\right)$ indicates $\delta=0$. Next, the sensitivity to $\theta_{12}$ was estimated only for $\delta=0$ and $\delta=\pi$ and is illustrated by three kinds of lines outside the colored. It is clear that the $\theta_{12}$ does not affect the fluxes as much as the $\theta_{23}$ does. The $\theta_{13}$ dependency in the fluxes is illustrated by the widths of the flux stripes. A remarkable improvement in precision of $\theta_{13}$ will be obtained from near

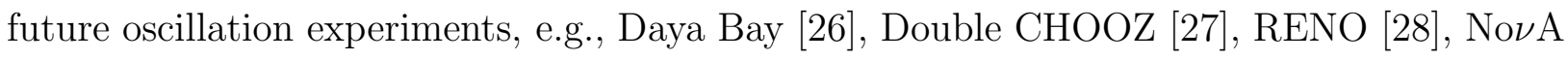


[29] and T2K [30]. In FIG. 1 and FIG. 2, two values of $\theta_{13}$ are used for comparison: 0.20 which is the current upper bound at $3 \sigma \mathrm{CL}$ and 0.082 which is the upper bound at $3 \sigma$ that RENO will accomplish. It is clearly illustrated that narrowing the stripes by a small bound of $\theta_{13}$ makes identifying the relative composition more realistic.

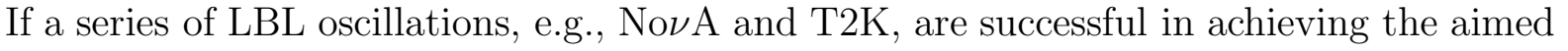
precision level, reducing the relative range of $\sin ^{2} \theta_{23}$ at $3 \sigma$ from $79 \%$ to $42 \%$ [31], the range in $\Phi^{t}\left(\nu_{e}\right)$ from the pure pion source is completely separated from the range in $\Phi^{t}\left(\nu_{e}\right)$ from the pure muon-damped source. The estimation of composition rate in a mixed beam of two pure sources requires the determination of $\mathrm{CP}$ phase $\delta$ as well as improved precisions of other parameters.

The curves of neutrino fluxes with respect to energy show both saturation and transition. For instance, the initial flux ratio $\Phi^{0}\left(\nu_{\mu}\right) / \Phi^{0}\left(\nu_{e}\right)$ of atmospheric neutrinos saturates to 2 at low energy limit [9] [10], while the expected ratio $\Phi^{t}\left(\nu_{\mu}\right) / \Phi^{t}\left(\nu_{e}\right)$ of high energy cosmic neutrinos to be detected saturates to 1 at low energy limit and to 1.8 at high energy limit [6]. If we can distinguish the saturation from the transition in flux - energy plots, the identification of neutrino beam from pure pion source or pure muon-damped source may be possible only after the accumulation of sufficient data of cosmic neutrino detection.

\section{LBL: A COMPLEMENT TO TELESCOPE}

The probability $P_{\mu e}$ in Eq. (15) depends on $\cos \left(\delta+\Delta m_{31}^{2} L / 4 E_{\nu}\right)$, while $\Phi^{t}(\nu)$ in Eq. (7) depends on $\cos \delta$. If $\Phi^{t}(\nu)$ and $P_{\mu e}$ are the orthogonal axes as in FIG. 3, the locus of $\Phi^{t}(\nu)$ and $P_{\mu e}$ for $\delta$ from 0 to $2 \pi$ completes a closed path. In FIG. 3, every locus in each figure passes a point in $P_{\mu e}-\Phi^{t}\left(\nu_{e}\right)$ space. The phrases $P\left(\nu_{\mu} \rightarrow \nu_{e}\right)$ and $\Phi\left(\nu_{\alpha}+\bar{\nu}_{\alpha}\right)$ in figures are equivalent to $P_{\mu e}$ and $\Phi\left(\nu_{\alpha}\right)$ in text. Especially in these figures, the probability $P_{\mu e}$ is an expected measurement of a super beam from J-PARC to Super-Kamiokande (T2K collaboration). Thus, input values are adopted from Ref. [30]. The baseline is $295 \mathrm{~km}$ and the energy of neutrino is $1 \mathrm{GeV}$. The matter effect $A$ in Eq. (5) is obtained with the Earth's density $2.8 \mathrm{~g} / \mathrm{cm}^{3}$ and the electron fraction 0.5. The Eq. (41) and Eq. (5) include only the leading terms. 

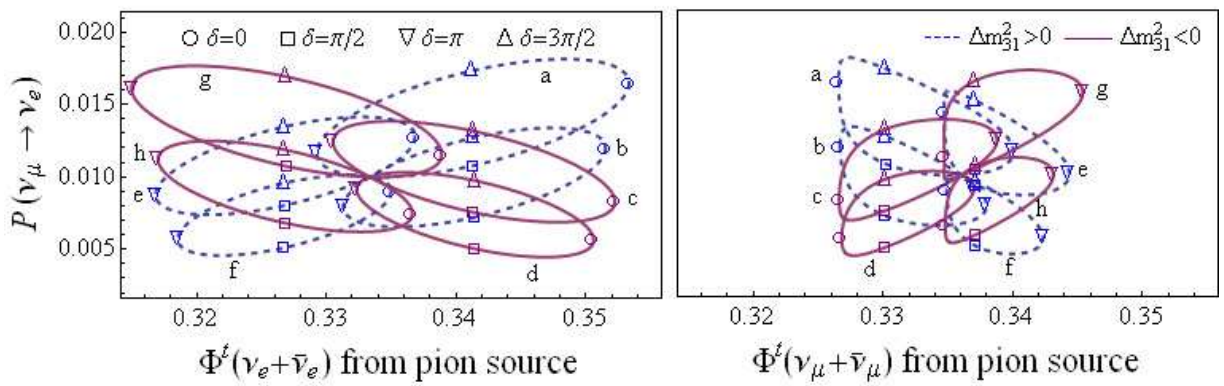

FIG. 3: Eight loci for $\delta \in[0,2 \pi]$ passing a common point in the $\Phi^{t}(\nu)-P_{\mu e}$ plane.

\section{A. Eightfold degeneracy}

A number of strategies are proposed to solve the degeneracy problems by using the results of LBL oscillations and reactor neutrino oscillations in future [19]-[24]. An example is to analyze long-baseline experiments over different oscillation distances [19], and another is to combine the results of reactor oscillations and the LBL [20]. This section checks possible resolutions obtainable from the combined analysis of results at telescopes and results at LBL.

The leading term of $P_{\mu e}$ in Eq. (5) with a remarkable sensitivity to $\theta_{13}$ does not distinguish $\Delta m_{31}^{2}>0$ from $\Delta m_{31}^{2}<0$ unless the matter effect is significant, and any detected flux $\Phi^{t}(\nu)$ is fully blind to $\Delta m_{31}^{2}$. However, the locus of $P_{\mu e}$ and $\Phi^{t}(\nu)$, as in FIG. 3 and FIG. 4, reveals the sign of mass-squared difference $\Delta m_{31}^{2}$ due to the phase difference $\Delta m_{31}^{2} L / 4 E_{\nu}$.

Each single curve in FIG. 3 is obtained by a set of specific values of $\theta_{23}, \theta_{12}$ and $\Delta m_{31}^{2}$, while $\delta$ runs from 0 to $2 \pi$. The value of $\theta_{12}$ is common for all curves in a figure and each of $\theta_{23}$ and $\Delta m_{31}^{2}$ has two choices: that $\theta_{23}$ may be a certain angle or its complementary angle and that $\Delta m_{31}^{2}$ may be $\left|\Delta m_{31}^{2}\right|$ or $-\left|\Delta m_{31}^{2}\right|$. Then, two sets of $\left(\theta_{13}, \delta\right)$ exist for every set of $\left(\theta_{23}, \Delta m_{31}^{2}\right)$, so that total eight pairs of $\left(\theta_{13}, \delta\right)$ 's describe the point in $P_{\mu e}-\Phi^{t}\left(\nu_{e}\right)[24]$. For instance, the point $\left(\Phi^{t}\left(\nu_{e}\right), P_{\mu e}\right)=(0.33,0.010)$ in FIG. 3 intersected by the eight curves can be specified by the following eight pairs of $\left(\theta_{13}, \delta\right)$ which belong to 'a - h'curves:

$$
\begin{array}{ll}
a:(0.104,2.3) & b:(0.087,3.8) \\
c:(0.094,2.4) & d:(0.079,3.6) \\
e:(0.086,0.86) & f:(0.071,5.6) \\
g:(0.103,1.01) & h:(0.084,5.4) .
\end{array}
$$


The above values of $\left(\theta_{13}, \delta\right)$ are divided into two groups, according to $\theta_{23}$ : [a, b, c, d] for $\theta_{23}=$ $\pi / 4-\epsilon$ and [e, f, g, h] for $\theta_{23}=\pi / 4+\epsilon$ where $\epsilon$ is a positive small angle. They can be divided also according to the sign of $\Delta m_{31}^{2}$ : [a, b, e, f] for $\Delta m_{31}^{2}>0$ and $[\mathrm{c}, \mathrm{d}, \mathrm{g}, \mathrm{h}]$ for $\Delta m_{31}^{2}<0$. In other words, the LBL oscillation probability $P_{\mu e}\left(\theta_{23}-\pi / 4, \operatorname{sgn}\left(\Delta m_{31}^{2}\right),\left(\theta_{13}, \delta\right)\right)=0.010$ is degenerated by the following eight possibilities

$$
\begin{aligned}
P_{\mu e}(-\epsilon,+, \mathrm{a}) & =P_{\mu e}(-\epsilon,+, \mathrm{b}) \\
= & P_{\mu e}(-\epsilon,-, \mathrm{c})=P_{\mu e}(-\epsilon,-, \mathrm{d}) \\
= & P_{\mu e}(+\epsilon,+, \mathrm{e})=P_{\mu e}(+\epsilon,+, \mathrm{f}) \\
= & P_{\mu e}(+\epsilon,-, \mathrm{g})=P_{\mu e}(+\epsilon,-, \mathrm{h}),
\end{aligned}
$$

where $P_{\mu e}(-\epsilon,+, a)$, as an example, means that the probability is obtained when $\theta_{23}-$ $\pi / 4=-\epsilon, \operatorname{sgn}\left(\Delta m_{31}^{2}\right)$ is + , and $\left(\theta_{13}, \delta\right)$ is the point on the locus 'a'. Likewise, the flux at telescope $\Phi^{t}\left(\nu_{e}\right)\left(\theta_{23}-\pi / 4, \operatorname{sgn}\left(\Delta m_{31}^{2}\right),\left(\theta_{13}, \delta\right)\right)=0.33$ is degenerated by the following eight possibilities

$$
\begin{aligned}
& \Phi^{t}\left(\nu_{e}\right)(-\epsilon,+, \mathrm{a})=\Phi^{t}\left(\nu_{e}\right)(-\epsilon,+, \mathrm{b}) \\
= & \Phi^{t}\left(\nu_{e}\right)(-\epsilon,-, \mathrm{c})=\Phi^{t}\left(\nu_{e}\right)(-\epsilon,-, \mathrm{d}) \\
= & \Phi^{t}\left(\nu_{e}\right)(+\epsilon,+, \mathrm{e})=\Phi^{t}\left(\nu_{e}\right)(+\epsilon,+, \mathrm{f}) \\
= & \Phi^{t}\left(\nu_{e}\right)(+\epsilon,-, \mathrm{g})=\Phi^{t}\left(\nu_{e}\right)(+\epsilon,-, \mathrm{h}) .
\end{aligned}
$$

Thus, a point representing data from two experiments, e.g., the point $\left(\Phi^{t}\left(\nu_{e}\right), P_{\mu e}\right)=$ $(0.33,0.010)$ or the point $\left(\Phi^{t}\left(\nu_{\mu}\right), P_{\mu e}\right)=(0.34,0.010)$ in FIG. 3, always belongs to many curves specified by different combinations of the parameters. The number of the loci passing a point represents the order of degeneracy. In fact, the order of degeneracy becomes infinite if $\Delta m_{31}^{2}$ or $\theta_{23}$ is allowed within a continuous range.

\section{B. Breakable degeneracy by high energy cosmic neutrinos}

FIG. 4 displays the loci of $P_{\mu e}$ and $\Phi^{t}(\nu)$ for $\theta_{12}$ from 0.51 to 0.69 and $\delta$ from zero to $2 \pi$ at $\theta_{13}=0.010$ and $\theta_{23}=\pi / 4$. The projections of the two bands onto $\Phi^{t}(\nu)$ axis overlap completely with each other, and even the projections of the two bands onto $P_{\mu e}$ axis overlap mostly with each other. So the individual measurement, either $\Phi^{t}(\nu)$ or $P_{\mu e}$, is not sensitive to the sign of $\Delta m_{31}^{2}$. However, the combination of two measurements as in FIG. 3 or FIG. [4 is significantly sensitive to whether $\Delta m_{31}^{2}>0$ or $\Delta m_{31}^{2}<0$. On the other hand, if $\theta_{23}$ 

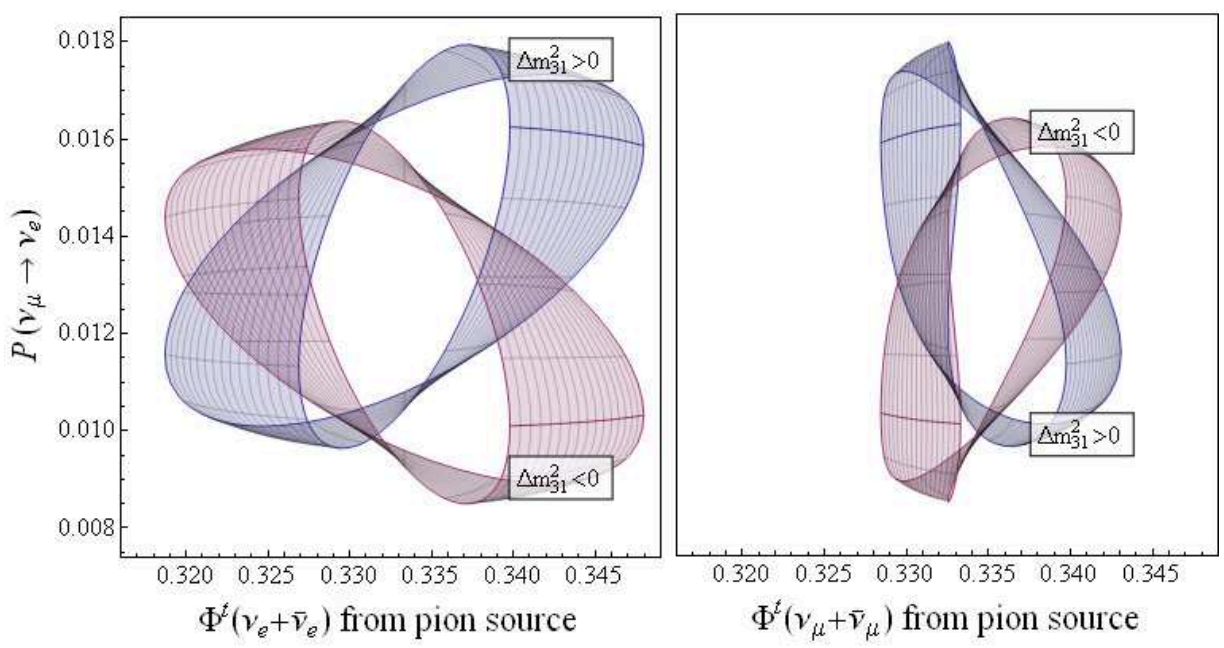

FIG. 4: Dependence of curves on $\Delta m_{31}^{2}: \theta_{12}$ is allowed within $3 \sigma$ CL.

and $\theta_{13}$ also are allowed within certain ranges rather than with fixed values, it is hard to get such distinct plots according to the type of mass hierarchy. Thus, an analysis with a fixed $\theta_{13}$ as in FIG. 4 can be effective only after a series of reactor or LBL neutrino oscillations, which will be launched ahead of neutrino detection at telescopes [26]-[30].

The leading term of $1-P_{\mu \mu}$ in Eq. (4) is sensitive to $\theta_{23}$ but does not distinguish $\theta_{23}=\pi / 4-\epsilon$ from $\theta_{23}=\pi / 4+\epsilon$. On the other hand, the flux $\Phi^{t}\left(\nu_{e}\right)$ in FIG. 5, which is more sensitive to $\theta_{23}$ than the probability $P_{\mu e}$ is, shows a distinction whether $\theta_{23}=\pi / 4-\epsilon$ or $\theta_{23}=\pi / 4+\epsilon$. Thus, $\Phi^{t}\left(\nu_{e}\right)$ which is a completely single-valued curve of $\theta_{23}$ in the currently allowed range as illustrated in FIG. 1 or in FIG. 5 avoids the degeneracy caused by the complementarity angle of $\theta_{23}$, unlike $1-P_{\mu \mu}$ in Eq. (4).

\section{Source identification}

FIG. 5 is drawn under the assumption which is that the neutrino beam was purely from the pion source and purely from the muon-damped source. In reality, the initial flux in an astronomical burst is difficult to identify, whether pion is produced in $p \gamma$ collision or $p p$ collision and whether the initial condition is pion source, muon-damped source, or a mixture of them when $p \gamma$ collision is dominant. Unlike the detection of photons at optical telescopes, the detection of neutrinos at telescopes should consider the change in neutrino flavors from the original outbursts. Moreover, broad uncertainties at present in neutrino mixing angles 

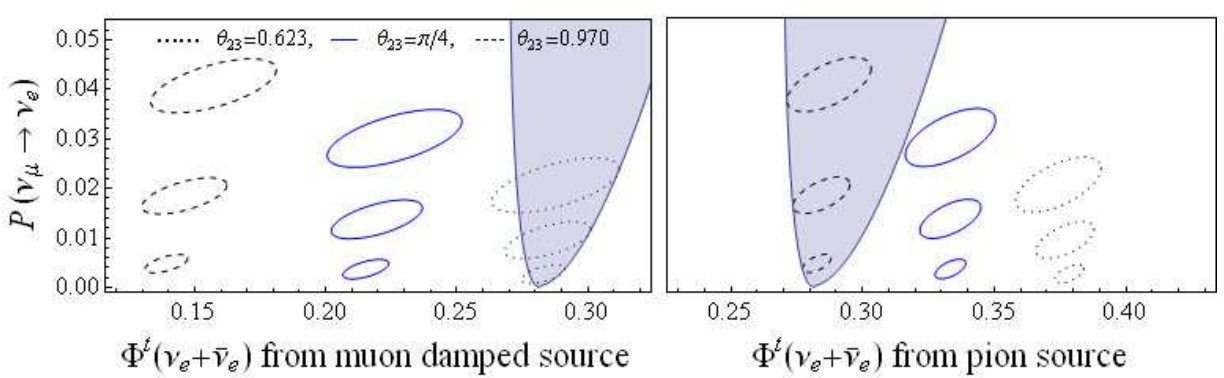

FIG. 5: The loci for $\delta \in[0,2 \pi]$ at $\theta_{13}=0.15,0.10$, or 0.05 for each $\theta_{23}$.
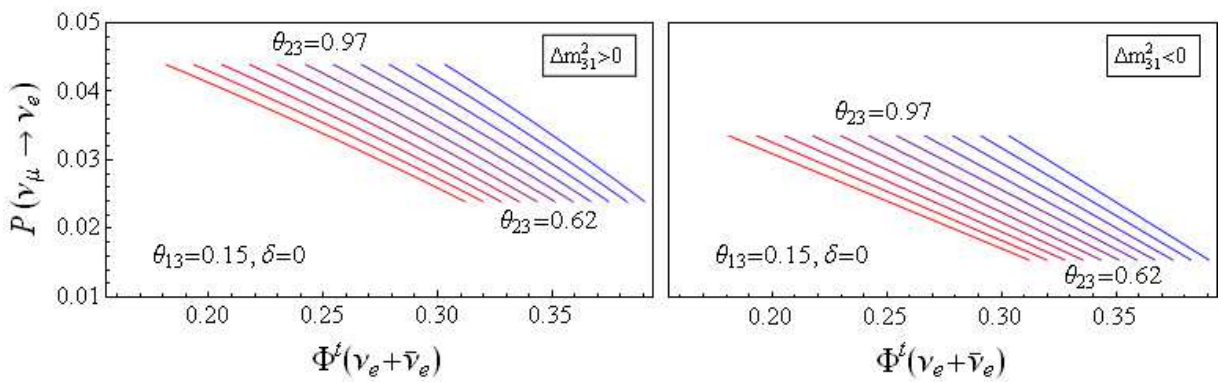

FIG. 6: The loci for $\theta_{23} \in[0.62,0.97]$ for various compositions.

obstruct telescopes in describing the initial condition of neutrino beams.

The LBL oscillation probability $P_{\mu e}$ may again share a role in specifying the source of cosmic neutrinos. The shaded regions of both panels in FIG. 5 show the same area which both sources cover commonly. So the measurement of $\Phi^{t}\left(\nu_{e}\right)$ within the shadow may indicate a beam from a pion source (right panel) for $\theta_{23}=0.970$ or a beam from a muon-damped source (left panel) for $\theta_{23}=0.623$. The distinction between them may be obtained by

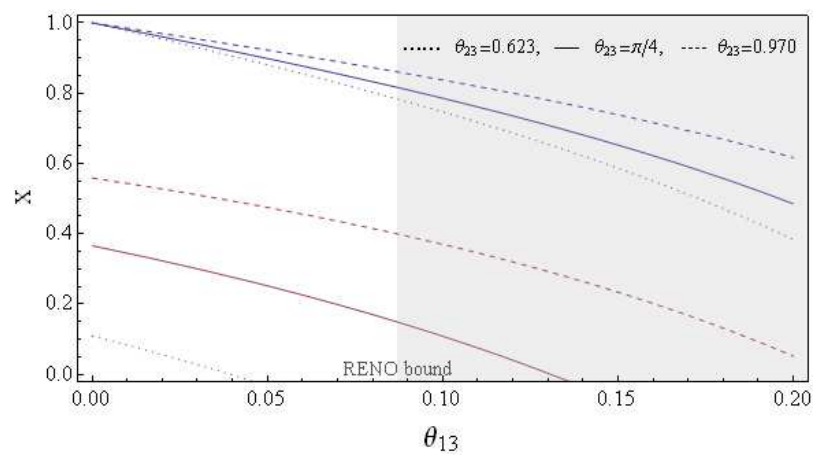

FIG. 7: The maximum portion of pion source in a mixed beam which can be distinguished from the pure pion source. 
considering $P_{\mu e}$ together with $\Phi^{t}\left(\nu_{e}\right)$. For instance, Fig. 5 tells us that $P_{\mu e}>0.025$ in the shadow can be compatible with $\Phi^{t}\left(\nu_{e}\right)$ from a pion source but not with $\Phi^{t}\left(\nu_{e}\right)$ from a muon-damped source.

FIG. [6] is an example that predicts the relative composition between a pion source and a muon-damped source, where $\theta_{12}=0.59, \theta_{13}=0.15$, and $\delta=0$. The change in color of the curves from the blue (rightmost) to the red (leftmost) indicate the change in composition from $100 \%$ pion source to $100 \%$ muon-damped source. Unfortunately, the distinction of the composition by every $10 \%$ as in Fig 6 lies far beyond the practicability, since current sensitivities of detectors are too low to use the strategy in the figure for the precise comparison.

The detected flux which is partially from pion source and partially from muon-damped source is expressed in terms of $x$, the portion of pion-source flux in the total detected $\nu_{e}$ flux

$$
\Phi^{t}\left(\nu_{e}\right)=x \Phi_{p}\left(\nu_{e}\right)+(1-x) \Phi_{m d}\left(\nu_{e}\right)
$$

where $\Phi_{p}$ is a flux from pion source and $\Phi_{m d}$ is a flux from muon-damped source. Even though the exact value of $\theta_{13}$ was known, the undetermined $\delta$ limits the composition of mixed flux or the pure pion-source flux to have blind ranges. FIG. 7 describes the maximum portion $X$ of $\Phi_{p}\left(\nu_{e}\right)$ in a mixed $\Phi^{t}\left(\nu_{e}\right)$ for various $\theta_{13}$ and $\theta_{23}$ that can be distinguished from the pure $\Phi_{p}\left(\nu_{e}\right)$, where $0<x<X$. The reason of decreasing $X$ as $\theta_{13}$ increases is because amplitudes of $\delta$ curves become more sizable. The upper(blue) lines are affected only by the range in $\delta$, while the lower(red) lines are affected by systematic uncertainties as well as by $\delta$. The sensitivity of IceCube detector to astrophysical source was discussed for neutrinos at $\mathrm{TeV}$ to PeV energies [32]. When $\frac{d N_{\nu}}{d E_{\nu}}$ is proportional to $E^{-2}$, the systematic uncertainty is $+10 /-15 \%$, and when $\frac{d N_{\nu}}{d E_{\nu}}$ is proportional to $E^{-3}$, the systematic uncertainty is $+5 /-20 \%$. In case of $E^{-2}$ spectrum, the composition of a distinguishable mixture from the pure pionsource beam is drawn by the lower (red) curves in FIG. 6. Within the current sensitivity of the IceCube detector, the mixed flux with more than $40 \%$ (or 20\%) pion source cannot be distinguished from the pure pion source when $\theta_{23}$ is 0.970 (or $\pi / 4$ ) and $\theta_{13}$ reaches the $3 \sigma$ upper bound at RENO. Within the current upper bound of $\theta_{13}$ as seen in the figure, the pure pion source cannot be distinguished even from the pure muon-damped source, unless $P_{\mu e}$ does help the identification in such a way as in FIG. 5 and FIG. 6. 


\section{CONCLUDING REMARKS}

Neutrino telescopes will detect neutrino beams out of astronomical bursts. However, the results to obtain are barely helpful in describing the initial condition of cosmic neutrino beams since the uncertainties in neutrino masses and mixing angles are broad and the fluxes to be measured are sensitive to the masses and the mixing angles. On the other hand, the improvement of the precision in parameters is also hard to attain by using results at neutrino telescope itself since even an original beam as initial condition cannot be defined without using the telescope.

We took a strategy to consider the fluxes to be detected at a telescope like IceCube in company with oscillation probabilities at a LBL T2K. The expected fluxes are examined for the sensitivities to mixing angles, in comparison with the sensitivities of other types of oscillation probabilities to mixing angles. A few restricted cases were presented as examples to show that neutrino fluxes at telescopes may be useful to resolve the degeneracies embedded in terrestrial neutrino oscillations. It was followed by the discussion on the limit of source identification which is allowed within the sensitivity of IceCube to astrophysical neutrinos.

\section{Acknowledgments}

K. Siyeon thanks Z. Xing for information on neutrino telescope and thanks physicists at IHEP in Beijing for warm hospitality. This work was supported by the Korea Research Foundation Grant funded by the Korean Government(MOEHRD).(KRF-2005-041-C00108)

[1] Q. R. Ahmad et al. [SNO Collaboration], Phys. Rev. Lett. 89, 011301 (2002)

[2] K. Hirata et al. [KAMIOKANDE-II Collaboration], Phys. Rev. Lett. 58, 1490 (1987).

[3] S. D. Wick, C. D. Dermer and A. Atoyan, Astropart. Phys. 21, 125 (2004)

[4] E. Waxman and J. N. Bahcall, Phys. Rev. Lett. 78, 2292 (1997)

[5] J. P. Rachen and P. Meszaros, Phys. Rev. D 58, 123005 (1998)

[6] T. Kashti and E. Waxman, Phys. Rev. Lett. 95, 181101 (2005)

[7] E. Waxman and J. Bahcall, Phys. Rev. D 59, 023002 (1998) 
[8] J. F. Beacom, N. F. Bell, D. Hooper, S. Pakvasa and T. J. Weiler, Phys. Rev. D 68, 093005 (2003) [Erratum-ibid. D 72, 019901 (2005)]

[9] T. K. Gaisser, T. Stanev and G. Barr, Phys. Rev. D 38, 85 (1988).

G. Barr, T. K. Gaisser and T. Stanev, Phys. Rev. D 39, 3532 (1989).

[10] M. Honda, K. Kasahara, K. Hidaka and S. Midorikawa, Phys. Lett. B 248, 193 (1990).

M. Honda, T. Kajita, K. Kasahara and S. Midorikawa, Phys. Rev. D 52, 4985 (1995)

[11] Y. Fukuda et al. [Kamiokande Collaboration], Phys. Lett. B 335, 237 (1994).

[12] Y. Fukuda et al. [Super-Kamiokande Collaboration], Phys. Lett. B 436, 33 (1998)

[13] J. Ahrens et al. [The IceCube Collaboration], Nucl. Phys. Proc. Suppl. 118, 388 (2003)

[14] T. Montaruli et al. [ANTARES Collaboration], arXiv:physics/0306057.

[15] J. G. Learned and S. Pakvasa, Astropart. Phys. 3, 267 (1995)

[16] H. Athar, M. Jezabek and O. Yasuda, Phys. Rev. D 62, 103007 (2000)

Z. Z. Xing and S. Zhou, Phys. Rev. D 74, 013010 (2006)

W. Winter, Phys. Rev. D 74, 033015 (2006)

K. R. S. Balaji, G. Couture and C. Hamzaoui, Phys. Rev. D 74, 033013 (2006)

D. Meloni and T. Ohlsson, Phys. Rev. D 75, 125017 (2007)

K. Blum, Y. Nir and E. Waxman, arXiv:0706.2070 [hep-ph].

W. Rodejohann, JCAP 0701, 029 (2007)

[17] W. M. Yao et al. [Particle Data Group], J. Phys. G 33, 1 (2006).

[18] M. Maltoni, T. Schwetz, M. A. Tortola and J. W. F. Valle, New J. Phys. 6, 122 (2004)

[19] V. Barger, D. Marfatia and K. Whisnant, Phys. Rev. D 65, 073023 (2002)

[20] H. Minakata, H. Sugiyama, O. Yasuda, K. Inoue and F. Suekane, Phys. Rev. D 68, 033017 (2003) [Erratum-ibid. D 70, 059901 (2004)]

[21] H. Minakata and H. Nunokawa, JHEP 0110, 001 (2001)

[22] G. L. Fogli and E. Lisi, Phys. Rev. D 54, 3667 (1996)

[23] J. Burguet-Castell, M. B. Gavela, J. J. Gomez-Cadenas, P. Hernandez and O. Mena, Nucl. Phys. B 608, 301 (2001)

[24] M. C. Gonzalez-Garcia and M. Maltoni, arXiv:0704.1800 [hep-ph].

A. Bandyopadhyay et al. [ISS Physics Working Group], arXiv:0710.4947 [hep-ph].

[25] G. Barenboim and C. Quigg, Phys. Rev. D 67, 073024 (2003)

[26] X. Guo et al. [Daya Bay Collaboration], arXiv:hep-ex/0701029. 
[27] F. Ardellier et al. [Double Chooz Collaboration], arXiv:hep-ex/0606025.

[28] K. K. Joo [RENO Collaboration], Nucl. Phys. Proc. Suppl. 168, 125 (2007). S. B. Kim [RENO Collaboration], AIP Conf. Proc. 981, 205 (2008).

[29] D. S. Ayres et al. [NOvA Collaboration], arXiv:hep-ex/0503053.

[30] Y. Itow et al. [The T2K Collaboration], arXiv:hep-ex/0106019.

[31] M. Lindner, Phys. Scripta T121, 78 (2005)

[32] J. Ahrens et al. [IceCube Collaboration], Astropart. Phys. 20, 507 (2004) 